\title{
DNS OF TURBULENT HEAT TRANSFER IN IMPINGING JETS AT DIFFERENT REYNOLDS AND PRANDTL NUMBERS
}

\section{MAGAGNATO F., SECCHI F., FOROOGHI P., STRAUB, S. AND FROHNAPFEL B.}

\author{
Institute of Fluid Mechanics (ISTM), Karlsruhe Institute of Technology (KIT), \\ Kaiserstr. 10, 76131 Karlsruhe, Germany \\ franco.magagnato@kit.edu / francesco.secchi@kit.edu
}

Key words: Impinging Jet, Turbulent Heat Transfer, Low Prandtl Number, SEM

\begin{abstract}
The heat transfer between an impinging circular jet and a flat plate is studied by means of direct numerical simulations (DNS) for different Prandtl numbers of the fluid. The thermal field is resolved for $P r=1,0.72,0.025$, and 0.01 . The flow is incompressible and the temperature is treated as a passive scalar field. The jet originates from a fully developed turbulent pipe flow and impinges perpendicularly on a smooth solid heated plate placed at two pipe diameters distance from the jet exit section. The values of Reynolds numbers based on the pipe diameter and bulk mean velocity in the pipe are set to $R e=5300$ and $R e=10000$. Inflow boundary conditions are enforced using a precursor simulation. Heat transfer at the wall is addressed through the Nusselt number distribution and main flow field statistics. At fixed Reynolds number it is shown that the Prandtl number influences the intensity of the Nusselt number at a given radial location, and that the Nusselt number distribution along the plate exhibit similar features at different Prandtl numbers. The characteristic secondary peak in the Nusselt number distribution is found for both Reynolds numbers for $P r=0.025$ and $P r=0.01$. All the simulations presented in this study were performed with the high order spectral element code Nek5000. Generated flow field statistics are available in the open access repository KITOpen.
\end{abstract}

\section{INTRODUCTION}

Fluid jets impinging on solid surfaces are commonly applied to a variety of industrial systems where heating, cooling, or drying of devices is needed. Gas turbine blades and electronic chip-sets are common examples in this respect. Jet impingement is also found in internal combustion engines, where the oxidant-fuel mixture is injected inside the combustion chamber and impinges on the surface of a moving piston. In almost all real-world applications impinging jets are turbulent with relatively large Reynolds numbers. Under these circumstances, the design process of devices exploiting impinging jets needs to rely heavily on models for the closure of Reynolds-averaged Navier Stokes equations (RANS). It is known that such models usually fail to predict, to a satisfactory degree, the main mean characteristics of heat transfer in the near-wall impingement region. In fact, Dairay et al. ${ }^{[1]}$ show that even some Large-Eddy-Simulation (LES) sub-grid models are challenged by this task. As a result, optimal designs of heat transfer systems are hardly achieved, with consequent tangible repercussions on the environmental and economic standpoint. For this reason, in the last decades a great deal of effort aimed at studying impinging jets experimentally and numerically. Reviews on the subject may be found in Livingood and Hrycak $^{[2]}$, Jambunathan et al. ${ }^{[3]}$, and Weigand and Spring ${ }^{[4]}$. Fundamental research on impinging jets in the past was mainly carried out via laboratory experiments, because of the unsustainable computational effort required for fully resolving such complex flow configuration in numerical simulations. In the work 
of Gardon and Akfirat ${ }^{[5]}$, experimental data are analysed focusing on the heat transfer characteristics along the impingement wall. For fully developed jets, i.e. jets originating from a fully developed flow condition, Reynolds number and nozzle to plate distance are the main parameters influencing the heat transfer distribution. For short jet lengths, higher Reynolds numbers lead to an increase in the global heat transfer while keeping the same shape of the mean heat transfer distribution. In the experimental work of Baughn and Shimizu ${ }^{[6]}$ the effects of the nozzle to plate distance on the heat transfer at the plate are tested for a single circular fully developed impinging jet at $R e=23750$. They show that the configuration with jet length equal to two diameters exhibits two distinct peaks in the local heat transfer radial distribution along the plate. The presence of two maxima in the heat transfer distribution is confirmed in the experimental investigation of Lee and Lee ${ }^{[7]}$ for the same jet length. In addition, they show that at higher Reynolds numbers the intensity of the two peaks is increased. For greater nozzle to plate distances, the second peak (i.e. the one further from the jet axis) eventually disappears.

With the technological advances in high-performance computing, fundamental research on impinging jets via numerical simulations became possible. Most of the existing literature is related to LES investigations on the dynamics of vortex structures and related effects on heat transfer enhancement. Single and multiple round and plane jets are considered in Beaubert and Viazzo ${ }^{[8]}$, Lodato et al. ${ }^{[9]}$, Hadžiabdić and Hanjalić ${ }^{[10]}$, Uddin et al. ${ }^{[11]}$, and Dairay et al. ${ }^{[1]}$. Conversely, only a few DNS studies are currently available. The work in Chung and Luo ${ }^{[12]}$ restricts the analysis to a 2-dimensional impinging jet up to $\operatorname{Re}=1000$ (based on the centerline velocity). On the other hand, Hattori and Nagano ${ }^{[13]}$ present DNS results of a plane jet at $\mathrm{Re}=9000$. The more recent study of Dairay et al. ${ }^{[14]}$ presents a fully resolved DNS of a circular impinging jet at $\mathrm{Re}=10000$. Their study aims at investigating the appearance of the aforementioned secondary maxinum in the mean $\mathrm{h}$
An extensive collection of statistics for different $\mathrm{R}$
of Wilke and Sesterhenn ${ }^{[5]}$. The huthors present
impinging on a flat surface at $\mathrm{Re}=3300$ and $\mathrm{Re}=$
Existent experimental and numerical investigati
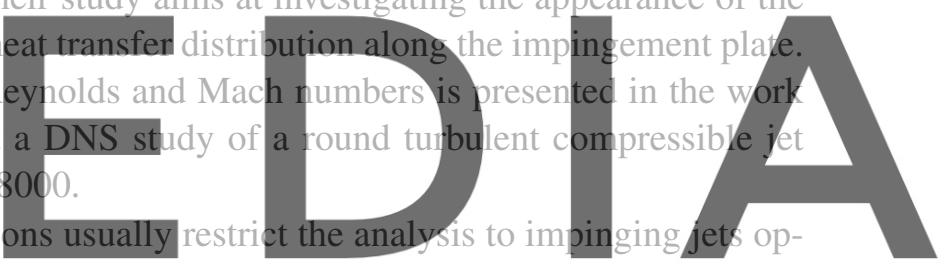

erating with fluids having a Prandtl number in the order of unity. Typically, air is used as working fluid

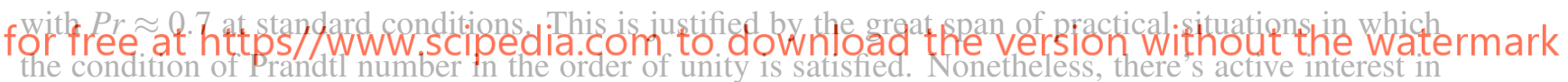

the prediction of heat transfer associated with low Prandtl number fluids. Liquid metals, such as liquid sodium (LS) or lead-bismuth eutectic (LBE), are for example considered as heat transfer fluid in central receiver systems which could increase their efficiency and make them economically more competitive. As shown in Pacio et al. ${ }^{[16]}$, thermal conductivity coefficients of LS and LBE are roughly one order of magnitude greater than other currently employed heat transfer fluids, such as molten nitrate salts. In this respect, LS and LBE exhibit also lower melting points and greater boiling temperatures, allowing to extend operating ranges at which they can be employed. Nonetheless, the introduction of liquid metals as heat transfer fluids in central receiver systems poses important challenges from the design perspective. At operating conditions, the Prandtl number of liquid metals is in the order of $0.025-0.001$. The turbulent heat transfer associated with low Prandtl number fluids is a highly complex phenomenon that is far from being well understood. Experimental assessment is indeed very demanding, and DNS studies are restricted to canonical flow configurations, such as channel flows (Kawamura et al. ${ }^{[17]}$, Piller et al. ${ }^{[18]}$, and Alcántara-Ávila et al. ${ }^{[19]}$ ), pipes, (Redjem-Saad et al. ${ }^{[20]}$ ), or backward-facing steps (Niemann and Frölich ${ }^{[21]}$ ).

In order to bridge this gap, the present work presents the DNS of a circular turbulent jet at $R e=5300$ 


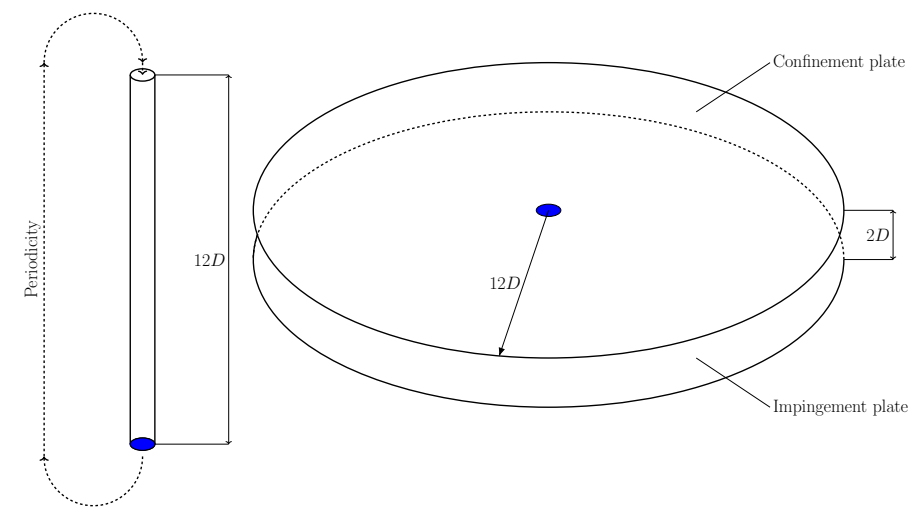

Figure 1: Schematic representation of the impinging jet flow configuration.

and $R e=10000$ impinging on a smooth solid heated wall placed at $2 D$ distance from the jet-nozzle exit. The flow is incompressible and the temperature field is treated as a passive scalar that diffuses and advects according to the local fluid velocity. The thermal field is resolved for Prandtl numbers $P r=1,0.72,0.025$, and 0.01 . A fully developed turbulent pipe flow simulation is used as a precursor for generating inflow boundary conditions for the jet. Despite the relatively high computational cost, the use of a precursor simulation allows to effectively impose a fully developed turbulent flow field at the inflow boundary of the computational domain. All the simulations in this study were performed using the open-source spectral element code Nek5000 ${ }^{[22]}$. Near-wall flow field statistics are available in the

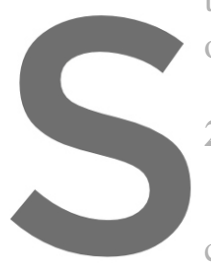
open access repository
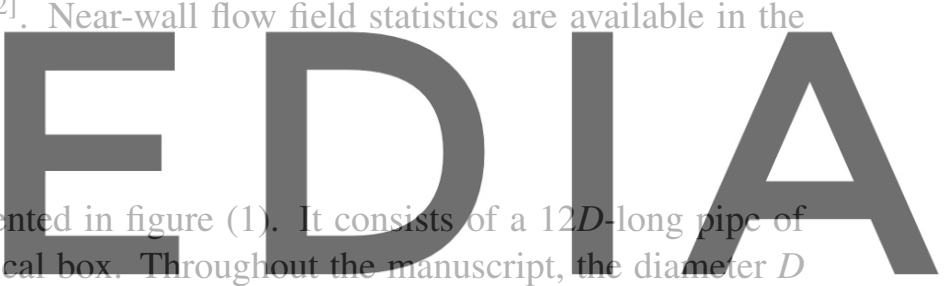

is used as reference length. The pipe is utilised to generate the jet inflow condition, while the cylindrical

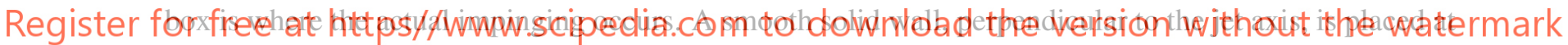
a distance $2 D$ from the jet exit section. A confinement plate, placed at the same height as the exit section of the jet, assures that no inflow of fluid into the computational domain is allowed from the sides of the jet. The outflow of the computational domain is represented by the lateral surface of the cylindrical box and it is placed at a distance $12 \mathrm{D}$ from the jet axis. Inflow boundary conditions at the jet exit section are generated using a precursor simulation strategy. In practice, a fully developed turbulent pipe flow with periodic boundary conditions is simulated concurrently with the simulation of the impinging jet. At each time step, the flow field at a given cross-section of the pipe is interpolated at the inflow section of the impinging jet simulation. Despite the high computational cost required, this technique allows to accurately reproduce a fully developed inflow for the jet. One should note that the flow condition at the pipe exit can significantly affect the wall heat flux (see e.g. Forooghi et al. ${ }^{[24]}$ ). This motivates use of a physical inflow condition in the present work despite the incurred cost. No-slip boundary conditions are applied for the velocity field on both the impingement and the confinement plate, while the outflow boundary condition of Dong ${ }^{[25]}$ is applied on the lateral surface of the computational box. A constant heat flux $q_{w}>0$ is applied on the impingement plate as boundary condition for the temperature field. All other surfaces of the computational domain are adiabatic, except the jet exit section which is kept at a 
constant temperature $T_{\text {jet }}$.

\section{NUMERICAL METHOD}

The flow under investigation is incompressible and free from the effect of any external volume force. The temperature field is treated as a passive scalar which is diffused and advected according to the local velocity field. Under these assumptions, the governing equations are the incompressible Navier-Stokes equations along with the equation(s) for the passive temperature field(s). Using tensorial notation, the system of equations in Cartesian coordinates reads:

$$
\begin{gathered}
\frac{\partial \mathbf{u}}{\partial t}+(\mathbf{u} \cdot \nabla \mathbf{u})+\nabla p-\frac{1}{R e} \nabla^{2} \mathbf{u}=0 \\
\nabla \cdot \mathbf{u}=0 \\
\frac{\partial T_{j}}{\partial t}+\mathbf{u} \cdot \nabla T_{j}=\frac{1}{\operatorname{Pr} R e} \nabla^{2} T_{j} \quad j=1,2, \ldots n_{\text {scal }}
\end{gathered}
$$

where $\mathbf{u}=(u, v, w)$ is the velocity of the fluid, $t$ is the time, $p$ is the pressure and $T_{j}$ (with $j=1,2, \ldots, n_{\text {scal }}$ ) is the $j^{\text {th }}$ passive scalar field. Equations (1) and (2) are made dimensionless using the bulk mean velocity $U_{b}$ in the pipe and the pipe diameter $D$. Thus, the Reynolds number is $\operatorname{Re}=U_{b} D / \mathrm{v}$, where $\mathrm{v}$ is the kinematic viscosity of the fluid, and the Prandtl number is $\operatorname{Pr}=\mathrm{v} / \alpha$, where $\alpha$ is the thermal diffusivity of the fluid. The numerical integration of equations (1) and (2) is performed using the open-source solver Nek5000[22]. The code $\mathrm{h}$ research groups around the by Maday and Patera ${ }^{[2}$ allelisation of its implementation equations in their weak form is $r$
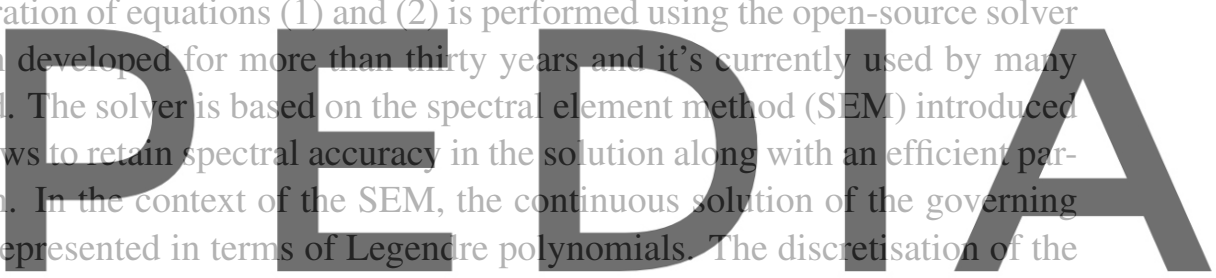

functional space of the solution and the test functions is achieved through the classical Galerkin approxi-

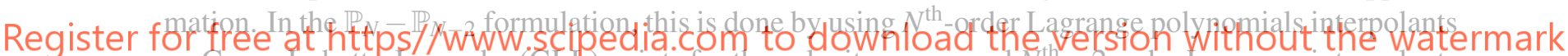
on Gauss-Lobatto-Legendre (GLL) points for the velocity space, and $N^{\text {th }}-2$ order Lagrange interpolants on Gauss-Legendre (GL) points for the pressure. In the Nek5000 framework it is also possible to rely on the $\mathbb{P}_{N}-\mathbb{P}_{N}$ formulation, where same order Lagrange interpolants are used for both velocity and pressure fields. In the present work only the $\mathbb{P}_{N}-\mathbb{P}_{N}$ has been applied. Time integration in Nek5000 is performed using an implicit-explicit scheme (BDFk-EXTk, backward differencing and extrapolation of order $k$ ). Namely, linear terms are treated implicitly, while non-linear terms are treated explicitly. Dealiasing of non-linear terms is realised using over-integration. The discretized problem is split into one Poisson problem for the pressure and one Helmholtz system for the velocity.

In the framework of Nek5000, equations (1) and (2) are are integrated numerically within a Cartesian coordinate system $(x, y, z)$. The origin is located at the intersection of the jet axis with the impingement plate. The $z$-axis coincides with the axis of the jet and it is oriented so as to measure the distance from the wall. $x$ - and $y$-axes lie on the plane of the impingement plate. Given the axial symmetry displayed by the flow configuration, during post-processing the solution and all statistical quantities accumulated during run-time are interpolated on a cylindrical coordinates mesh whose coordinate system has the origin and $z$-axis coinciding with the original Cartesian coordinate system. Within the new coordinate system, $r$ denotes radial locations, $\theta$ measures rotations, and $z$ measures the distance from the 
Magagnato F., Secchi F., Forooghi P., Straub S. and Frohnapfel B.

Table 1: Parameters for the impinging jet simulations

\begin{tabular}{cccc}
\hline Case & Order of solution & Number of grid points & $\Delta z_{\text {wall,max }}^{+}$ \\
\hline $\operatorname{Re}=5300$ & $5^{\text {th }}$ & $\approx 616 \mathrm{M}$ & $\approx 0.4$ \\
\hline $\operatorname{Re}=10000$ & $5^{\text {th }}$ & $\approx 616 \mathrm{M}$ & $\approx 0.6$
\end{tabular}

Table 2: Parameters for the precursor pipe flow simulations. In the table $R$ represents the pipe radius

\begin{tabular}{ccccccc}
\hline Case & Order & N. of grid points & $R e_{\tau}$ & $\Delta r_{\text {wall }}^{+}$ & $\Delta z^{+}(\min -\max )$ & $R \Delta \theta^{+}(\min -\max )$ \\
\hline$R e=5300$ & $3^{r d}$ & $\approx 70 \mathrm{M}$ & $\approx 182$ & $\approx 0.4$ & $\approx 2.3-6.2$ & $\approx 1.3-2.4$ \\
\hline$R e=10000$ & $3^{\text {rd }}$ & $\approx 70 \mathrm{M}$ & $\approx 316$ & $\approx 0.7$ & $\approx 4.1-10$ & $\approx 2.2-4.1$
\end{tabular}

impingement plate. The vector velocity field is denoted by the radial component $u_{r}$, azimuthal component $u_{\theta}$, and axial component $u_{z}$.

\section{RESULTS}

The flow under investigation is statistically invariant under rotation around the jet axis. For this reason, flow variables can be averaged, other than in time, also in the azimuthal direction. The resultant averaged flow field is two-dimensional, as an averaged variable depends on the radial position from the jet axis, $r$, and the distance from the impingement plate, $z$. In what follows, $\langle\cdot\rangle$ will be used to denote averaging in both time and azimuthal direction. After reaching the statistically steady state, flow

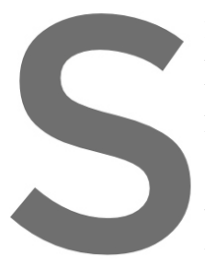
field statistics have been Dairay et al. ${ }^{[14]}$ estimates frequency of large scale statistics corresponds to 20 the impinging jet are sum collected in time for 50 external time unit
a natural Strouhal number $\$ t=0.4$ (based
structures in the jer. With this assumptio
cycles of large eddies impingement. Parar
marized in table (1). Figure (2) displays the
$\mathbf{\Delta z})^{1 / 3}$ and the Kolmogorov length scale $\eta=$
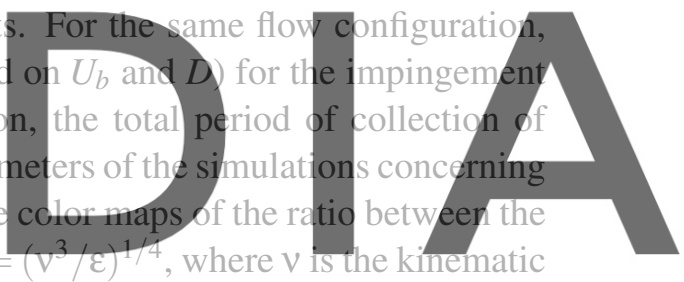
local grid size $\Delta=(\Delta x, \Delta z)$ a a viscosity of the fluid, and $\varepsilon$ is the dissipation of turbulent kinetic energy. For the $R e=5300$ case, figure

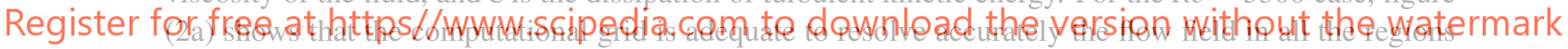

of interest of the computational domain. In fact, in the near-wall impingement region, it can be seen that $\Delta \leq 3 r$. The same computational grid is also used at $R e=10000$, thus resulting in a lower resolution of the flow field. From figure (2b), it can be seen that the resolution in the near-wall impingement region can still be considered reasonable also in this case. A grid convergence analysis with a lower order solution ( $3^{\text {rd }}$ order) has shown that the maximum difference between the computed mean Nusselt number distributions at $R e=10000$ is always lower than $2 \%$ for $r / D<3.5$. Table (2) reports parameters regarding the precursor pipe flow simulations. For both Reynolds numbers, the grid resolution in the pipe has been kept fixed not to increase excessively the computational overhead. Nonetheless, main pipe-flow statistics agree well with reference data in the literature. Figure (3) shows the comparison of the computed mean streamwise velocity and Reynolds shear stress profiles with data from Khoury et al. ${ }^{[27]}$ for pipe flow at $R e=5300$ and $R e=11000$.

\subsection{Mean velocity field}

Color maps of mean axial and radial velocity components are reported in figure (4), while figure (5) represents color maps of root mean square turbulent fluctuations $u_{r, r m s}, u_{z, r m s}$, and $u_{\theta, r m s}$. In the figures, 


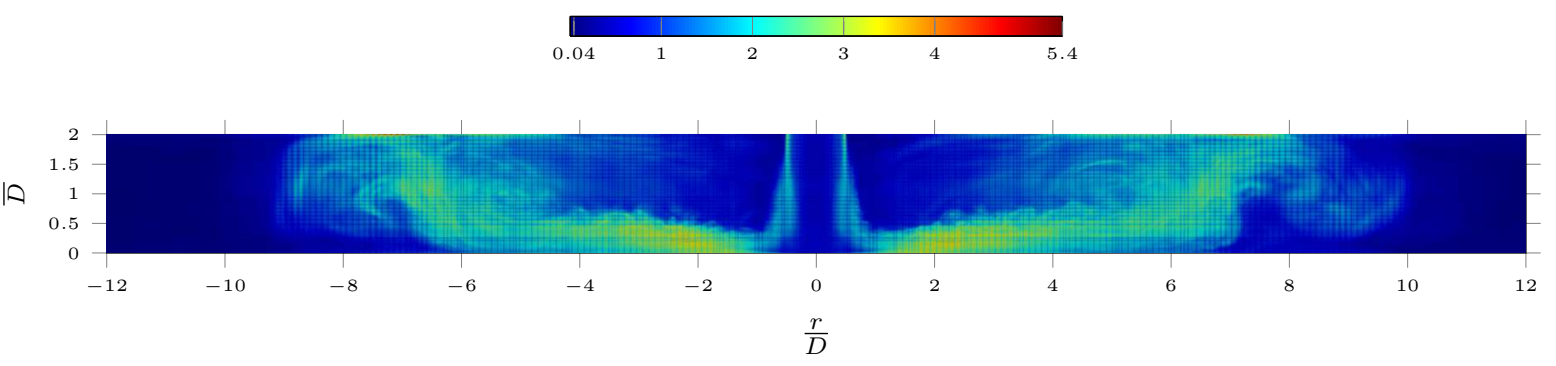

(a)

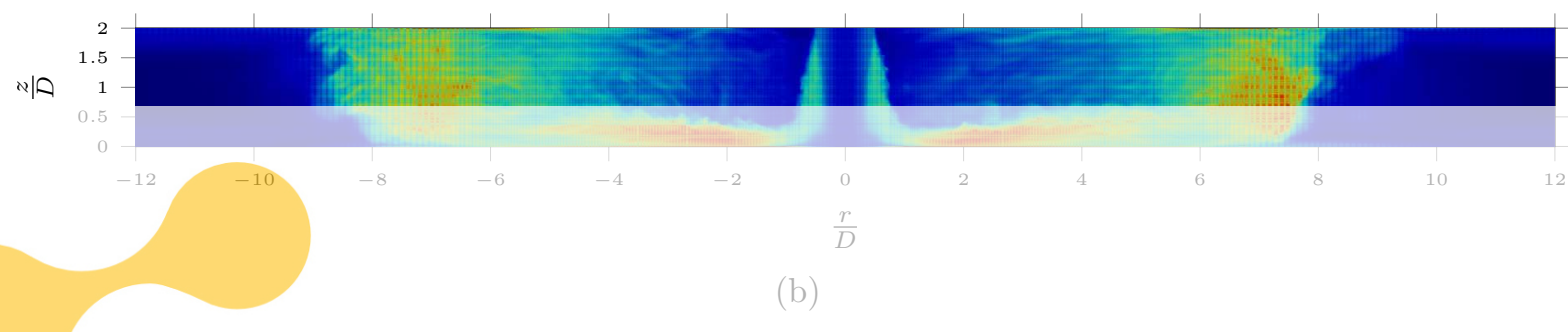

Figure 2: Maps of the ratio of local grid size $(\Delta x \Delta y \Delta z)^{1 / 3}$ to Kolmogorov length scale $\eta$. (a) $R e=5300$; (b) $R e=10000$.

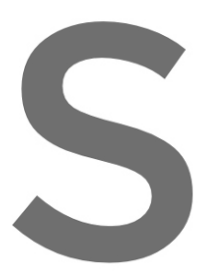

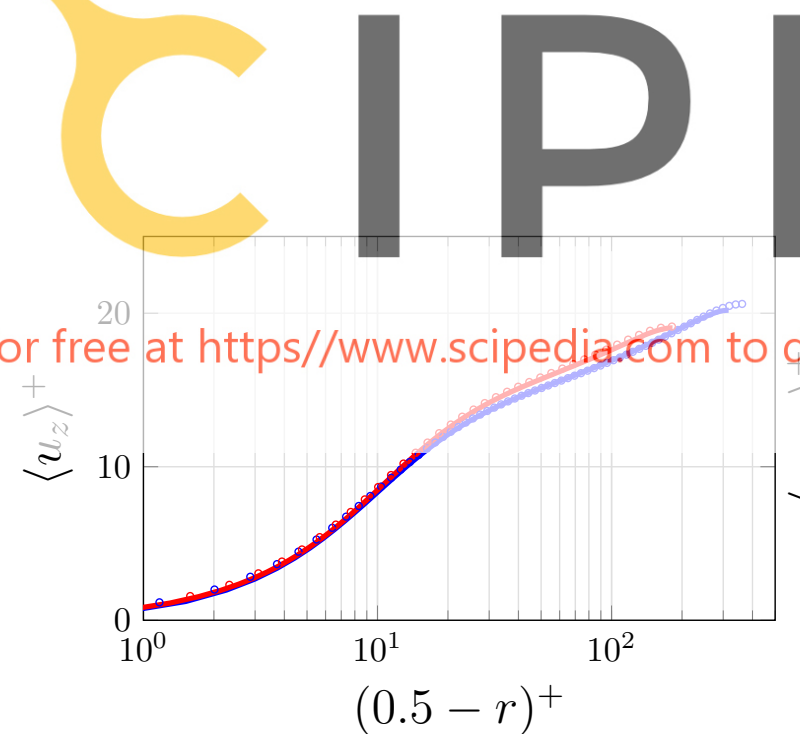

(a)

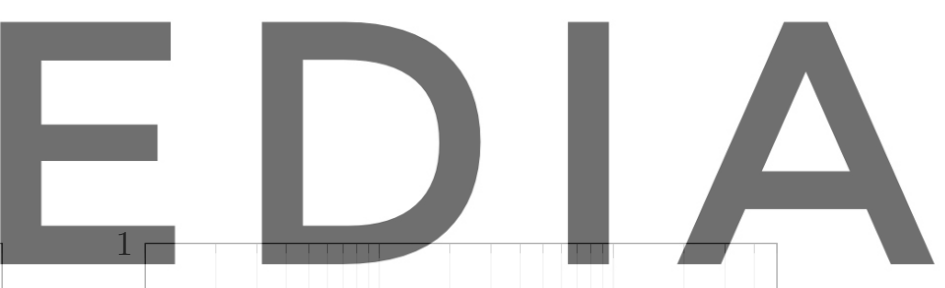

Register for free at https//www.scipedia.eom to download the version without the watermark

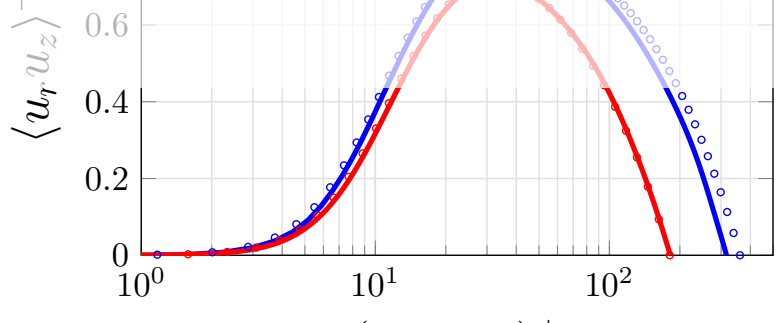

$(0.5-r)^{+}$

(b)

Figure 3: Main flow statistics in viscous units for the precursor pipe flow simulations. Reference data from Khoury et al. ${ }^{[27]}$ $\circ \circ, R e=5300 \circ \circ \circ, R e=11000$. Present DNS: , $R e=5300$ , $R e=10000$. (a) Mean axial velocity $\left\langle u_{z}\right\rangle^{+}$; (b) Reynolds shear stress component $\left\langle u_{r} u_{z}\right\rangle^{+}$. In the figure, the abscissa $(0.5-r)^{+}$measures the distance from the wall in viscous units. 


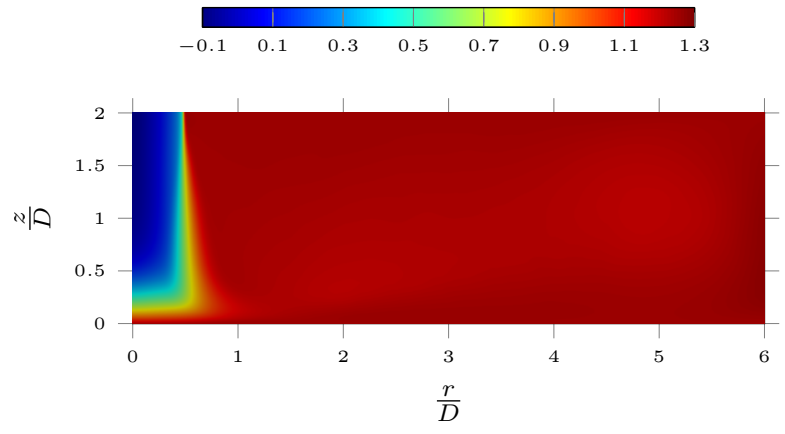

(a)

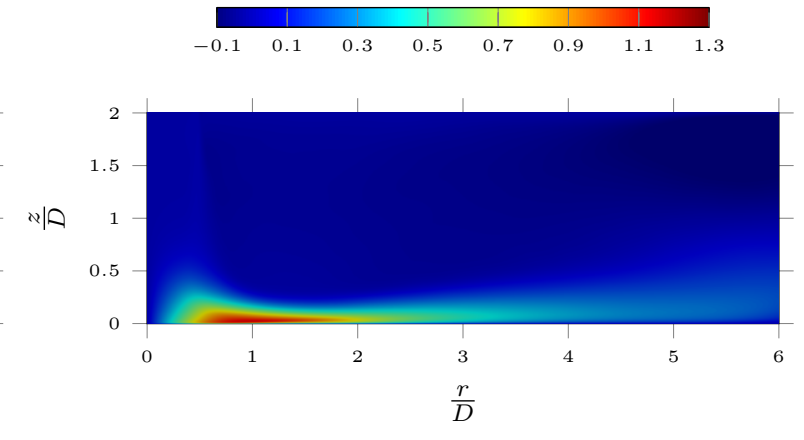

(b)

Figure 4: Color maps of the averaged velocity field at $R e=10000$. (a) Axial component, $\left\langle u_{z}\right\rangle$; (b) radial component, $\left\langle u_{r}\right\rangle$.

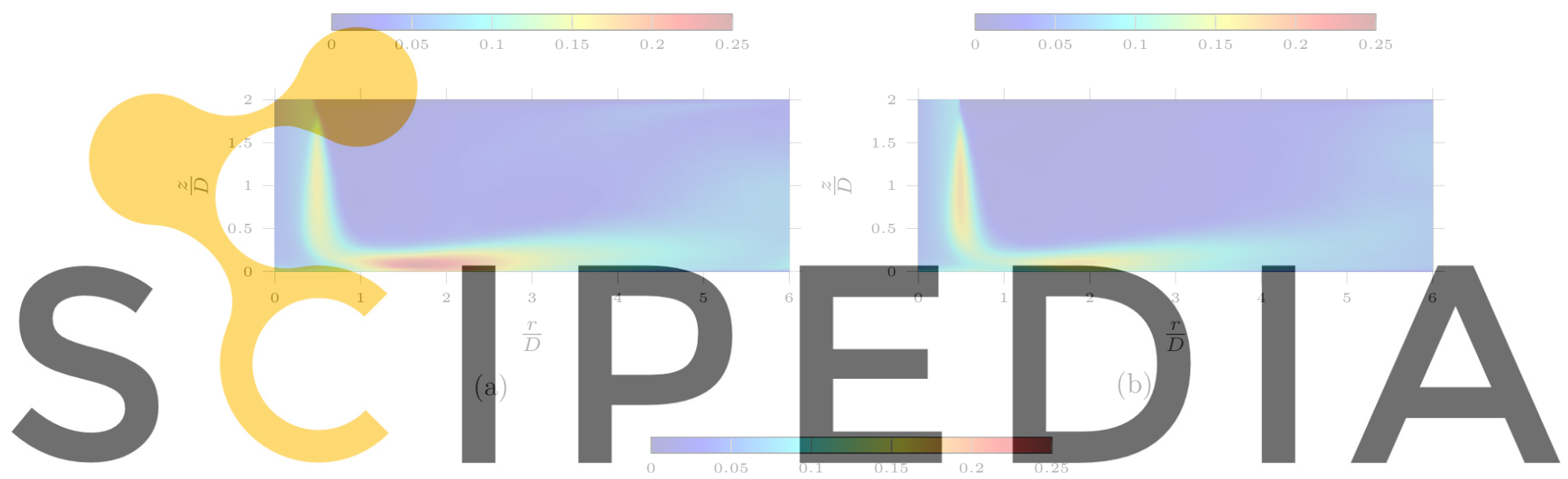

Register for free at https//www.scipedia.com to download the version without the watermark

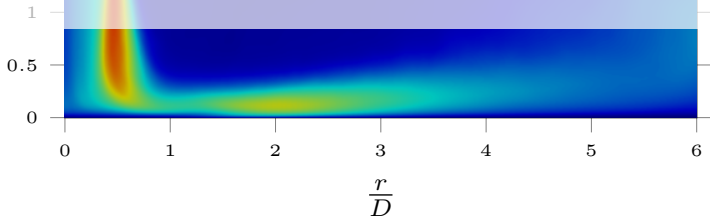

(c)

Figure 5: Color maps of the root mean square turbulent fluctuations at $R e=10000$. (a) Radial component, $u_{r, r m s}$; (b) azimuthal component, $u_{\theta, r m s}$; (c) axial component, $u_{z, r m s}$

only mean flow statistics for the $R e=10000$ case are reported, but similar observations can be drawn also for $R e=5300$. From the analysis of the figures, characteristic flow regions can be distinguished. A potential core, characterized by very low turbulent fluctuations and a predominant axial velocity, exists for $z>0.6 D$ and $r<0.25 D$. The so-called potential core region is a typical feature of free-jets and it is also exhibited by impinging jets, provided that the nozzle to wall distance is large enough. Moving 
towards the impingement plate, the mean velocity gradually deflects and becomes parallel to the radial direction. This region of the jet is usually referred to as stagnation region and, in the present case, it covers $0<r / D<1$ and $0<z / D<0.5$. In the stagnation region, both the axial and radial velocity components have a significant magnitude. Moving away from the stagnation region, the mean velocity becomes completely aligned with the radial direction. Here a turbulent boundary layer develops and gets thicker as the fluid slows down due to friction losses at the wall and mixes with the adjacent quiescent fluid. This region, which extends for $r / D>1.5$, is usually termed the wall-jet region.

\subsection{Mean Heat transfer}

A measure of the heat transfer along the impingement wall is the mean Nusselt number distribution. This is defined as

$$
\langle N u\rangle(r)=\frac{q_{w} D}{\lambda\left(\left\langle T_{w}\right\rangle(r)-T_{j e t}\right)}
$$

where $\lambda$ represents the thermal conductivity of the fluid and $\left\langle T_{w}\right\rangle$ is the mean wall temperature at a distance $r$ from the jet axis. The mean Nusselt number distribution along the plate is displayed in figure (6) for both Reynolds numbers and $\operatorname{Pr}=1$, and $\operatorname{Pr}=0.72$. Comparison is made with reference DNS data of Dairay et al. ${ }^{[14]}$ for $R e=10000$ and $\operatorname{Pr}=1$ and with experimental data of Lee and Lee ${ }^{[7]}$ for $R e=10000, R e=5000$ and $\operatorname{Pr}=0.7$. Figure (6a) shows the Nusselt distributions for the $R e=10000$ case. The overall agreement with DNS data of Dairay et al. ${ }^{[14]}$ is very good, except for a different prediction of the stagnation Nusselt number (i.e. $N u$ at $r / D=0$ ), and for minor deviations observed

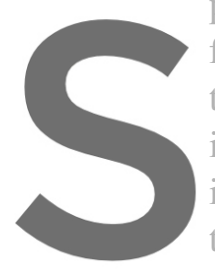
for $r / D>2.5$. The former can be attributed to d
to differences in the outflow treatment. Regardin
investigation of Forooghi et al. ${ }^{224} \mathrm{~s}$ shows that, for a
in the present study, inflow boundary conditions h
transfer characteristics. In the work of Dairay et a
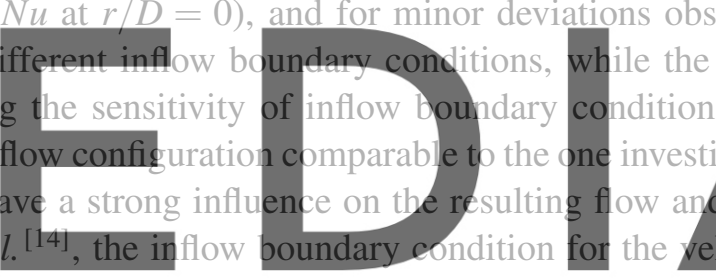

consists of a prescribed velocity profile at the jet exit section. Turbulence is then triggered in the jet

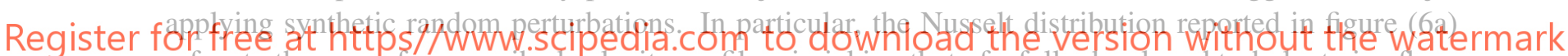
refers to the case of a prescribed velocity profile mimicking that of a fully developed turbulent pipe flow.

This is different from what is done in the present work, where an actual fully developed turbulent flow field is interpolated at the inlet section of the computational domain. On the other hand, deviations in the Nusselt number distribution for $r / D>2.5$ can be caused by different strategies used for the outflow boundary treatment. In the present work, the outflow boundary is placed at $12 D$ distance from the jet axis and the stabilized boundary condition of Dong ${ }^{[25]}$ is applied there. On the other hand, in the work of Dairay et al. ${ }^{[14]}$ the domain extends up to $6 D$ and a fringe method is used. The agreement with experimental data of Lee and Lee ${ }^{[7]}$ is especially good for the lower Reynolds number case displayed in figure (6b). At $R e=10000$, figure (6a), the experimental Nusselt distribution shows two distinct local peaks at $r / D \approx 0.5$ and $r / D \approx 1.5$. The calculated distribution is maximum at the jet axis $(r / D=0)$ and it has a secondary peak at $r / D \approx 2$. This shape for the Nusselt number distribution, and especially the appearance of the secondary peak, is well documented in the literature for the same flow configuration. On the other hand, as extensively reported in Dairay et al. ${ }^{[14]}$, two distinct peaks characterise the Nusselt number distribution in the case of a non-fully developed turbulent flow field at the inflow of the jet. At lower Prandtl numbers, the overall shape of the Nusselt number distribution along the plate remains unchanged. This fact is shown in figure (7), which depicts the Nusselt number distributions for $\operatorname{Pr}=$ 


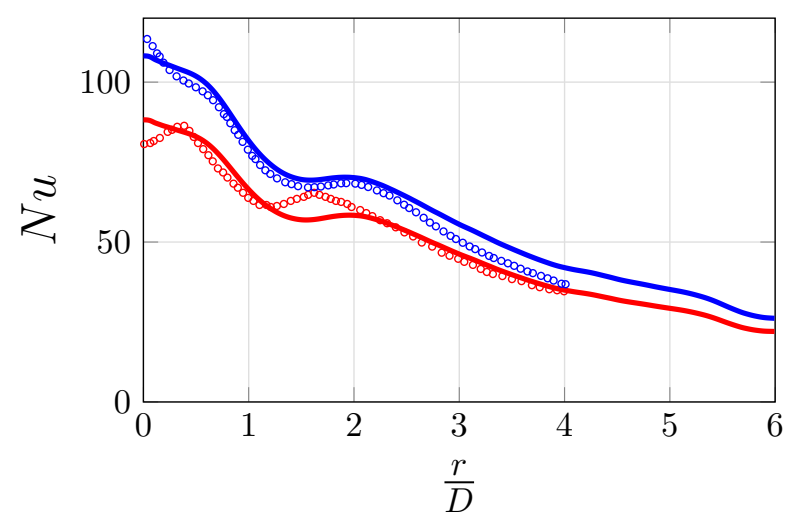

(a)

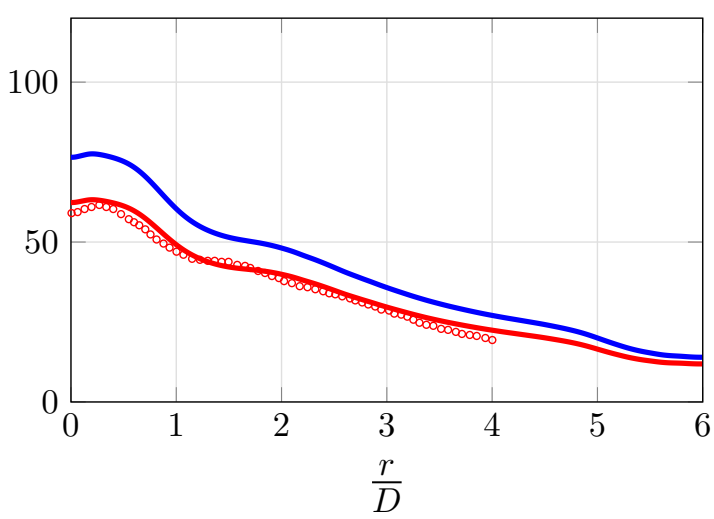

(b)

Figure 6: Mean Nusselt number distribution along the impingement plate. Reference data from Lee and Lee ${ }^{[7]}$ : $\circ \circ \circ, \operatorname{Pr}=0.7$; reference data from Dairay et al. ${ }^{[14]}: \circ \circ \circ, \operatorname{Pr}=1$. Present DNS: $=, P r=1 ;-, P r=0.72$. (a) $\operatorname{Re}=10000$; (b) $R e=5300$ present results, and $R e=5000$ reference data.

0.025 and $\operatorname{Pr}=0.01$ at $R e=10000$ (figure (7a)) and $R e=5300$ (figure (7b)). In the stagnation region, the Nusselt number attains a global maximum value for all Prandtl numbers and then decreases moving away from the jet axis. For $\mathrm{Pr}=0.01$, the decrease in Nusselt number for increasing radial distance is less pronounced with respect to larger Prandtl numbers cases at the same Reynolds number. In particular,

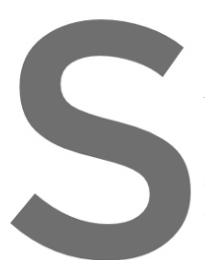
at $R e=5300$, the Nusselt distribution displays an alm $R e=10000$, a local mini same feature is observed only for $P r=0.025$ and distribution is evidenced at $r / D \approx 2$ for all the case lower values of the Prandtl number, the peak can
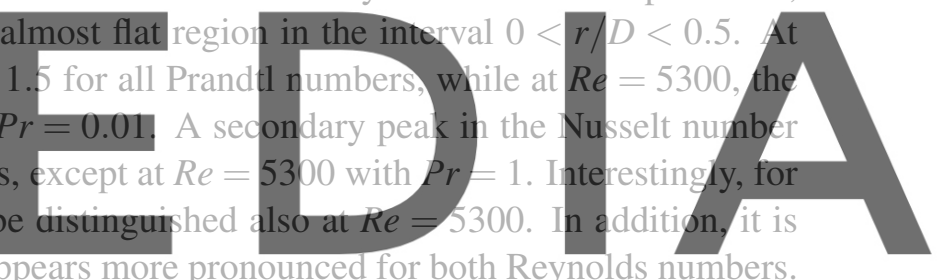

observed that for $\operatorname{Pr}=0.025$, the secondary peak appears more pronounced for both Reynolds numbers.

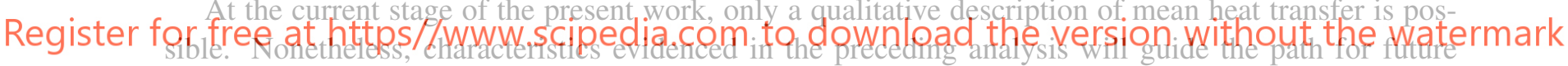

investigations. Immediate work will mainly focus on the analysis of the large data-set generated in the course of this study. In particular, budget equations for the turbulent kinetic energy, mean temperature field and variance of the temperature field will be assessed in different regions of the flow domain. This will aid the phenomenological investigation of turbulent heat transfer in the near-wall region and provide turbulence modeling research groups with a rich collection of flow field statistics yet not available in the literature.

\section{CONCLUSIONS}

The present work presented DNS results of a turbulent jet impinging on a flat solid heated wall. Concurrent simulations of fully developed turbulent pipe flow at $R e=5300$ and $R e=10000$ were used to feed the inlet section of the jet. The use of a precursor simulation to generate inflow boundary conditions represents a novelty in the context of the DNS of impinging jets and allows to accurately reproduce realistic inflow conditions that are usually met in experiments. The temperature field was treated as a passive scalar and it was resolved for different values of the Prandtl number of the fluid. In addition to 


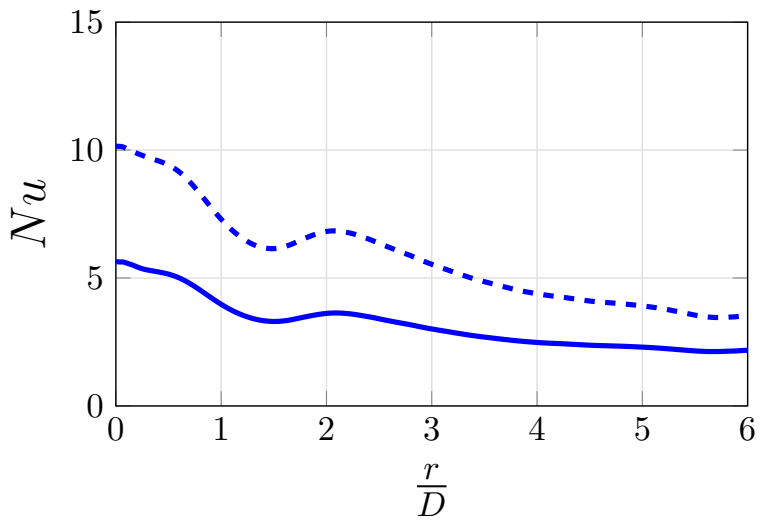

(a)

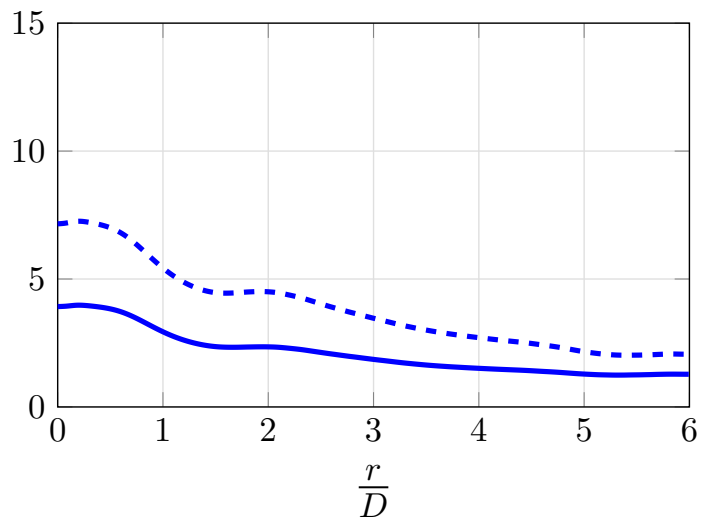

(b)

Figure 7: Mean Nusselt number distribution along the impingement plate. $=-=-=\operatorname{Pr}=0.025 ; \quad \square \operatorname{Pr}=0.01$. (a) $R e=10000$; (b) $\operatorname{Re}=5300$.

classical values of $\operatorname{Pr}=1$ and $\operatorname{Pr}=0.72$, which are already extensively documented in the literature, the present investigation reported for the first time results for $\operatorname{Pr}=0.025$ and $\operatorname{Pr}=0.01$. The heat transfer at the impingement wall was addressed through a description of the mean flow field. A thorough understanding of the phenomena involved in the heat transfer enhancement associated with impinging show indeed that the dyn the mean heat transfer mean flow quantities, for heat transfer. In particular distribution along the ilmpingement plate. As exp
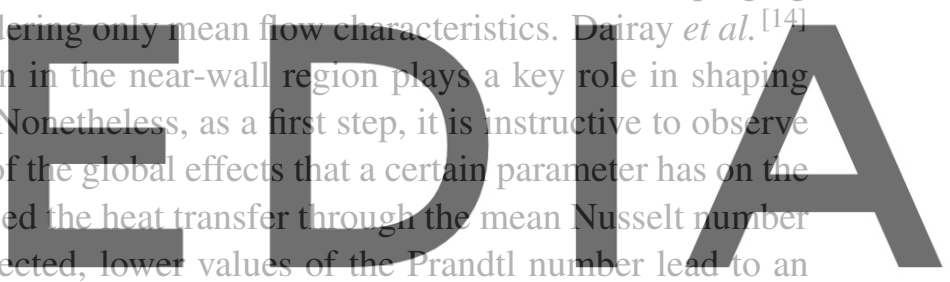
overall decrease in the Nusselt number at each radial location along the plate. As a result, the cooling

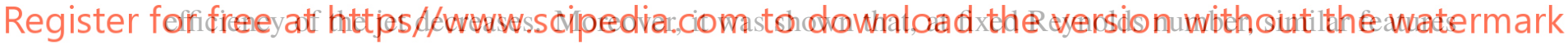
characterise the mean Nusselt number distribution for all Prandtl numbers. At Re $=10000$, the Nusselt distribution features a secondary peak at $r / D \approx 2$ for all Prandtl numbers investigated. Interestingly, for $P r=0.025$ the peak appears more pronounced in comparison to the other cases. Similar observations were drawn also at $R e=5300$, with the notable difference that the secondary peak was observed only for $\operatorname{Pr}=0.025$ and $\operatorname{Pr}=0.01$.

\section{Acknowledgement}

Support by the German Research Foundation (DFG) under Collaborative Research Centre SFB/ TRR150 project B02 is greatly acknowledged.

The simulations were performed on the national supercomputer HPE Apollo Hawk at the High Performance Computing Center Stuttgart (HLRS) under the grant number 21196. 


\section{References}

[1] F. Dairay, V. Fortuné, E. Lamballais, and L. E. Brizzi. LES of a turbulent je impinging on a heated wall using high-order numerical schemes. Int. J. Heat Fluid Flow, 50:177-187, (2014).

[2] J. N. B. Livingood and P. Hrycak. Impingement heat transfer from turbulent air jets to flat plates: a literature survey nasa, lewis research center. NASA Technical Memorandum, (1973).

[3] K. Jambunathan, E. Lai, M. A. Moss, and B. L. Button. A review of heat transfer data for single circular jet impingement. Int. J. Heat Fluid Flow, 13(2):106-115, (1992).

[4] B. Weigand and S. Spring. Multiple jet impingement - a review. Heat Transfer Res., 42(2):101$142,(2011)$.

[5] R. Gardon and J. C. Akfirat. The role of turbulence in determining the heat-transfer characteristics of impinging jets. Int. J. Heat Mass Transf, 8(10):1261-1272, (1965).

[6] J. W. Baughn and S. Shimizu. Heat transfer measurement from a surface with uniform heat flux and an impinging jet. J. Heat Transfer, 111:1096-1098, (1989).

[7] J. Lee and S. J. Lee. Stagnation region heat transfer of a turbulent axisymmetric jet impingement. Exp. Heat Transfer, 12(2):137-156, (1999).

[8] F. Beaubert and S. Viazzo. Large eddy simulations of plane turbulent impinging jets at moderate reynolds numbers. Int. J. Heat Fluid Flow, 24(4):512-519, (2003).

[9] G. Lodato, L. Vervisch, and P. Domingo. A compressible wall-adapting similarity mixed model for large-eddy simulation of the impinging round jet. Phys. Fluids, 21(3):035102-1 - 035102-21, (2009).

[10] M. Hadžiabdić and K. Hanjalić. Vortical structures and heat transfer in a round impinging jet. $J$. Fluid Mech., 596:221-260, (2008).

[11] N. Uddin, S. O. Neumann, and B. Weigand. Les simulations of an impinging jet: on the origin of the second peak in the nusselt number distribution. Int. J. Heat Mass Transf, 5(1):356-368, (2013).

[12] Y. Chung and K. H. Luo. Unsteady heat transfer analysis of an impinging jet. J. Heat Transfer, 124(6):1039-1048, (2002).

[13] H. Hattori and Y. Nagano. Direct numerical simulation of turbulent heat transfer in plane impinging jet. Int. J. Heat Fluid Flow, 25(5):749-758, (2008).

[14] F. Dairay, V. Fortuné, E. Lamballais, and L. E. Brizzi. Direct numerical simulation of a turbulent jet impinging on a heated wall. J. Fluid Mech., 764:362-394, (2015).

[15] R. Wilke and J. Sesterhenn. Statistics of fully turbulent impinging jets. J. Fluid Mech., 825:795824, (2017).

[16] J. Pacio, A. Fritsch, C. Singer, and R. Uhlig. Liquid metals as efficient coolants for high-intensity point-focus receivers: Implications to the design and performance of next-generation csp systems. Energy Procedia, 49:647-655, (2014).

[17] H. Kawamura, K. Ohsaka, H. Abe, and K. Yamamoto. DNS of turbulent heat transfer in channel flow with low to medium-high prandtl number fluid. Int. J. of Heat and Fluid Flow, 19:482-491, 
(1998).

[18] M. Piller, E. Nobile, and T.J. Hanratty. DNS study of turbulent transport at low prandtl numbers in a channel flow. J. of Fluid Mech., 458:419-441, (2002).

[19] F. Alcántara-Ávila, S. Hoyas, and M.J. Pérez-Quiles. DNS of thermal channel flow up to re=2000 for medium to low prandtl numbers. Int. J. of Heat and Mass Transfer, 127:349-361, (2018).

[20] L. Redjem-Saad, M. Ould-Rouiss, and G. Lauriat. Direct numerical simulation of turbulent heat transfer in pipe flows: Effect of prandtl number. Int. J. of Heat and Fluid Flow, 28:847-861, (2007).

[21] M. Niemann and J. Fröhlich. Buoyancy-affected backward-facing step flow with heat transfer at low prandtl number. Int. J. Heat Mass Transfer, 101:1237-1250, (2016).

[22] P.F. Fisher, J.W. Lottes, and S.G. Kerkemeier. NEK5000 Version 19.0. https://nek5000.mcs. anl.gov/. Argonne National Laboratory, Illinois.

[23] F. Secchi and F. Magagnato. DNS of turbulent heat transfer in impinging jets at different Reynolds and Prandtl numbers. DOI: 10.5445/IR/1000129589.

[24] P. Forooghi, B. Frohnapfel, and F. Magagnato. Simulation of a gaseous jet impinging on a convex heated surface-effect of inlet condition. Appl. Therm. Eng., 105:1076-1084, (2016).

[25] S. Dong. A convective-like energy-stable open boundary condition for simulations of incompressible flows. J. Comput. Phys., 302:300 - 328, (2015).

[26] Y. Maday and A.T. Patera. Spectral element methods for the incompressible Navier-Stokes equations. In State of the art surveys on computational mechanics ASME, pages 71-143, (1989).

[27] G. Khoury, P. Schlatter, A. Noorani, P. Fischer, G. Brethouwer, and A. Johansson. Direct numerical simulation of turbulent pipe flow at moderately high reynolds numbers. Flow, Turbulence and Combustion, 91:475-495, (2013). 